Relations industrielles

Industrial Relations

\title{
La FTQ et l'action politique provinciale
}

\section{Roger Provost}

Volume 13, numéro 1, janvier 1958

URI : https://id.erudit.org/iderudit/1022465ar

DOI : https://doi.org/10.7202/1022465ar

Aller au sommaire du numéro

\section{Éditeur(s)}

Département des relations industrielles de l’Université Laval

\section{ISSN}

0034-379X (imprimé)

1703-8138 (numérique)

Découvrir la revue

\section{Citer ce document}

Provost, R. (1958). La FTQ et l'action politique provinciale. Relations industrielles / Industrial Relations, 13(1), 54-57.

https://doi.org/10.7202/1022465ar
Résumé de l'article

Voici le texte du discours prononcé par M. Roger Provost, président, à l'ouverture du 2ième congrès annuel régulier de la Fédération des Travailleurs du Québec. On sait que la position de M. Provost sur les attitudes politiques de la FTQ fut ultérieurement ratifiée par le congrès, qui eut lieu les 14, 15 et 16 novembre 1957.
Tous droits réservés @ Département des relations industrielles de l’Université Laval, 1958
Ce document est protégé par la loi sur le droit d'auteur. L'utilisation des services d'Érudit (y compris la reproduction) est assujettie à sa politique d'utilisation que vous pouvez consulter en ligne.

https://apropos.erudit.org/fr/usagers/politique-dutilisation/ 


\title{
COMMENTAIRES
}

\section{LA FTQ ET L'ACTION POLITIQUe A L'ECHELLE PROVINCIALE}

\section{Roger Provost}

\begin{abstract}
Voici le texte du discours prononcé par M. Roger Provost, président, à l'ouverture du 2ième congrès annuel régulier de la Fédération des Travailleurs du Québec. On sait que la position de $M$. Provost sur les attitudes politiques de la FTQ fut ultérieurement ratifiée par le congrès, qui eut lieu les 14, 15 et 16 novembre 1957.
\end{abstract}

Nous en sommes au premier congrès annuel régulier de la Fédération des Travailleurs du Québec. Mes premiers mots seront pour souhaiter la bienvenue aux délégués venus des quatre coins de la Province pour juger les actes de leurs officiers au cours des huit derniers mois et participer à la détermination de la politique de votre Fédération pour les mois qui viendront.

Plusieurs activités ont marqué les derniers mois. Il serait oiseux ici d'énumérer ce qu'a fait votre Fédération depuis la dernière conférence. La plupart d'entre vous ont participé personnellement à ces activités. Ceux qui n'ont pu le faire ont trouvé dans leurs journaux les commentaires et les reportages qui ont suivi les gestes que nous avons posés. Nul ne peut maintenant mettre en doute le «militantisme » de votre centrale provinciale. Le point culminant qui a donné naissance aux démonstrations dont nous avons été les auteurs et les acteurs fut la négation presque complète du droit d'association pour plusieurs ouvriers de cette Province, notamment ceux de Murdochville. Il est regrettable de constater qu'au 20ème siècle, dans une Province où la démocratie est censée exister, il faille encore entreprendre des marches de protestation pour éveiller la population au fait que ce droit est foulé aux pieds en certain cas. Ce qui est plus déplorable encore, c'est qu'après que des milliers de citoyens se sont fait tuer sur les champs de bataille pour protéger la liberté, des ouvriers trouvent la mort en temps de paix en luttant pour la liberté d'association.

Le droit d'association est un droit naturel et un droit sacré. Le nier, c'est nier que l'être humain est un être sociable, c'est nier aux membres d'une société la liberté de s'unir pour défendre leurs droits, leurs opinions, leurs croyances. La négation des droits sur le plan social ou économique entraîne tôt ou tard la négation de la liberté politique, et c'est le chemin le plus rapide vers la dictature avec ce qu'elle a d'inhumain, d'anti-social et même d'anti-chrétien.

Depuis des années, nous présentons des mémoires aux gouvernements de cette Province afin que la liberté d'association soit garantie. Les quelques amendements aux différentes lois ouvrières que nous avons réussi à décrocher au cours des années n'ont pu améliorer la situation, puisqu'ils ont été annihilés par d'autres amendements et aussi par l'interprétation que les corps constitués ont donné à la même loi.

Au cours de la dernière année, des groupes d'ouvriers majoritaires constituant quelque huit mille individus se sont vu refuser le droit d'association pour des raisons pseudo-légales ou pour d'autres raisons qui n'en n'étaient pas. Il est malheureux que nos cours de justice, apparemment en guerre contre les commissions administratives, se soient plu, afin paraît-il de réaffirmer les droits judiciaires, à distribuer des brefs de prérogative à droite et à gauche à qui les voulait. Les seules victimes de cette rivalité apparente ont été les ouvriers. Les seuls bénéficiaires ont été les patrons qui refusent de reconnaître les libertés fondamentales de ceux qu'ils emploient et leurs responsabilités sociales comme chefs d'entreprise. Le cas de Murdochville, où les tribunaux ont pris un an à décider d'un bref de prérogative, 
n'est pas le seul; et nous pourrions en citer d'autres, moins spectaculaires peut-être, où après 14 mois la cause n'a pas même été entendue. Tous ceux qui sont concernés savent quels sont les remèdes à apporter au code civil et à la Loi des Relations Ouvrières, mais personne ne semble intéressé à promouvoir ces amendements. Y aurait-il connivence en haut lieu, ou bien les intéressés seraient-ils simplemet désintéressés? Au cours des prochains mois, il nous faudra trouver les moyens d'éclairer la population tout entière à ce sujet, afin de créer une opinion publique assez puissante pour forcer les responsables à donner aux ouvriers le droit d'association sans ambages et sans restrictions.

Nul ne peut nier que l'être humain est libre de vendre son travail à qui il veut et quand il le veut. Il peut donc ainsi refuser de travailler lorsqu'il le décide pour des raisons qu'il convient à lui seul de juger. Afin de prévenir les cataclysmes ou les soubresauts économiques que pourraient engendrer des grèves trop fréquentes et trop nombreuses, l'Etat a jugé bon d'entourer l'exercice de ce droit de certaines restrictions. A cette fin, on a institué des tribunaux de conciliation et d'arbitrage et on a établi certaines conditions à l'exercice du droit de grève. Nous ne nous objectons pas aux tribunaux de conciliation qui ont pour fonction d'amener les deux parties à conclure une entente et à assurer la paix industrielle, pourvu que ces tribunaux soient constitués à cette fin. Cependant, lorsque les tribunaux de conciliation ou d'arbitrage deviennent simplement des tribunaux d'enquête ou pis encore lorsqu'ils deviennent des institutions dominées par la politique et qu'ils se font les instruments de destruction des syndicats par des méthodes dilatoires, i] nous faut nous demander si la conciliation obligatoire est une solution et un gage de paix industrielle, ou si elle n'est pas plutôt une chambre noire où les syndicats qui par un heureux hasard ont obtenu la reconnaissance syndicale sont soumis à une mort lente mais certaine. La conciliation doit être volontaire, et les parties devraient avoir le privilège de s'en prévaloir ou ne pas s'en prévaloir avant la déclaration de la grève ou de la contre-grève. De cette façon, des efforts réels seraient faits par les parties à la conciliation pour en arriver à la signature d'une convention de travail. De plus, aussi longtemps que les parties en cause (et nous savons qu'elles le désirent dans plusieurs cas) ne pourront choisir des présidents qui ne soient pas des créatures politiques, sur des listes établies par les associations patronales et syndicales et par les groupes universitaires, les hommes de bonne foi, qu'ils soient des représentants patronaux ou syndicaux, mettront en doute même la validité de leur présence.

Afin d'attirer l'attention de l'opinion publique sur ce qu'il advient de certains droits fondamentaux en cette province, nous avons eu recours à l'action directe, qui s'est révélée en mème temps quelque peu spectaculaire, d'autant plus que ce genre d'action était nouveau en cette province. De là, on s'est demandé avec quelque inquiétude quelle position adoptait ou allait prendre le mouvement ouvrier québecois. Certains voyaient un mouvement ouvrier tout d'abord politique; d'autres craignaient même que le mouvement ouvrier allait chambarder l'ordre établi et tenter de tout mener à sa guise. Les institutions allaient être boulevêrsées. A tel point qu'à un certain moment, des groupes patronaux confortablement installés dans des «clubs exclusifs » ont pensé à une contre-offensive.

Il est incontestable que le mouvement ouvrier constate de plus en plus qu'il lui faut dépasser les cadres classiques de la lutte exclusivement économique, et ceci pour deux raisons. Tout d'abord, il est de plus en plus conscient (et les événements qui se sont produits ces derniers mois ont réveillé pleinement cette conscience), que le facteur politique intervient davantage dans le développement et l'épanouissement économiques du mouvement ouvrier. Son existence, son progrès, son avancement sont conditionnés par les lois passées par des parlements et administrées par des commissions désignées par ces mêmes parlements. L'incidence politique est donc importante.

En second lieu, la responsabilité du mouvement ouvrier, sa fonction dans une société démocratique dépasse le champ de la revendication économique, i.e. de la négociation et de la surveillance des conventions. Le syndiqué est un citoyen, et comme tel, sans faire ressusciter les luttes de classes, il doit se reconnaître une 
responsabilité collective dans le domaine politique. Il doit tendre à maintenir la démocratie dans sa forme la plus pure, là où elle existe encore comme telle, à s'efforcer de restaurer les normes démocratiques là où elles sont menacées, à donner un sens à la politique en créant une véritable conscience politique chez ses compagnons et concitoyens.

Nous devons néanmoins conserver comme premier objectif la revendication économique. et éviter de passer d'une abstention désastreuse à une action échevelée qui ne se veut que spectaculaire et qui ne renferme rien de positif ni de concluant. Le remède alors pourrait devenir pire que le mal lui-même et serait susceptible de conduire à la destruction du mouvement syndical en cette Province.

Que doit être alors l'action politique qui pourrait remplir les deux buts énumérés plus haut? Me serait-il permis de vous dire d'abord que la formule adoptée ne pourrait en être une pour chaque province sans considération de la politique générale du Congrès du Travail du Canada en la matière. Les prochains mois nous amèneront peut-être à des prises de positions nouvelles sur le plan national qui offriraient des cadres au sein desquels notre action pourrait s'orienter sur le plan provincial.

Certains pourraient opiner pour la fondation d'un parti à l'échelle provinciale. Que voulez-vous, nous avons la réputation dans cette province d'être des fondateurs. Toutefois, à ceux-là je dirai qu'un tel parti dans le contexte actuel n'a aucune chance de succès. Tout d'abord, à cause de la carte politique, même s'il est favorisé, il ne pourrait être qu'un faible groupe minoritaire. En second lieu, vous admettrez avec moi qu'une éducation poussée s'impose avant que nous puissions convaincre nos membres d'appliquer les recommandations des corps centraux ou même d'emboîter le pas derrière eux. J'ai ici en mémoire les dernières élections complémentaires provinciales, notamment dans le comté de Compton.

D'autres veulent à tout prix se venger de ceux qui sont en place, à cause de leurs attitudes et de leurs gestes anti-syndicaux. J'ai pour ceux-là la plus grande sympathie, je souhaite comme eux un changement et pour les mêmes raisons. Mais l'histoire politique du monde nous a montré qu'il est dangereux de provoquer des changements profonds dans une fureur négative à moins d'être prêts à redonner des cadres qui soient sûrement nôtres.

Il me semble donc qu'il nous faudra tout d'abord créer une conscience politique non seulement chez nos ouvriers industriels mais aussi chez les ouvriers agricoles, les cultivateurs, afin que l'action politique, lorsqu'elle deviendra électorale, ait des chances de succès et ait en même temps un caractère essentiellement positif. Education politique, oui mille fois oui! Education poussée, consistante. au niveau de l'union locale, au niveau du Conseil du Travail, au niveau de la Fédération, en coopération avec les autres groupes économiques, sociaux, universitaires, mille ${ }^{*}$ fois oui, et immédiatement.

Action politique sous forme de démonstrations populaires afin d'amener des prises de position de la part du public en général, oui mille fois oui.

Mais partout, cette éducation et cette action doivent conserver un caractère positif et surtout ne pas détruire ni faire passer en second lieu la fonction principale du mouvement ouvrier qui est la revendication économique. Tout est à bâtir sur le plan politique pour le mouvement ouvrier. Le besoin est là, le désir est présent, mais ceux qui ont accepté des resnonsabilités ont le devoir d'ériger ce nouvel édifice sur des bases solides et de penser aux assises encore plus qu'à la façade.

Je ne voudrais pas prolonger indéfiniment ce colloque, mais je fais appel à tous les délégués, quelles que soient leurs craintes de s'engager dans ce qui pour eux constitue l'inconnu dangereux ou quels que soit la hâte et le désir qu'ils aient de devenir le facteur déterminant de la politique en cette province, de ne pas laisser dominer exclusivement leur moi, leur désir ou leur philosophie indivi- 
duelle, mais de considérer l'ensemble du mouvement ouvrier. En dépit de certains articles de journaux, trop spéculatifs à notre avis, notre mouvement doit sortir de cette conférence plus uni, plus déterminé, plus militant que jamais.

Vous avez la responsabilité de décider de l'avenir de plusieurs milliers d'ouvriers dans nos unions locales. Cette responsabilité dépasse nos opinions personnelles, dépasse les préjugés que nous pourrions avoir. Je sais que vous vous montrerez à la hauteur de ces graves responsabilités. Que ces trois jours puissent marquer d'une pierre blanche l'histoire du mouvement ouvrier de cette province.

\section{LA CTCC ET L'AFFILIATION AU CTC}

\section{GÉrard Dion}

La question des rapports à établir entre la CTCC et le CTC intéresse à juste titre tout le public canadien. Nous avons déjà retracé dans le numéro spécial de Relations Industrielles sur la fusion CTMC-CCT les attitudes officielles prises par la CTCC en ces dernières années. ${ }^{1}$

Comme le congrès de 1956 s'était prononcé en faveur du principe de l'affiliation de la CTCC au CTC et qu'une attitude définitive devrait être prise au cours d'un congrès spécial qui aurait lieu au plus tard le ler juin 1957, on s'attendait que le congrès annuel de 1957 apporterait quelque chose de nouveau. Or il n'en n'a été à peu près rien.

Tout d'abord le congrès spécial a été décommandé parce qu'une question préalable n'avait pu être réglée: celle de la réforme des structures internes de la CTCC.

La question a cependant rubondi devant le congrès. Deux résolutions venant de groupes particuliers s'opposaient à l'affiliation. L'une d'elle, celle de la Fédération du bâtiment (numéro 68) ne donnait aucun considérant. L'autre, celle de la Fédération des employés des corporations municipales et scolaires (numéro 69), se présentait comme suit:

«Considérant que l'affiliation (ou la fusion) est un problème complexe; Considérant que le Comité de l'Unité syndicale n'a pas terminé son étude; Considérant que la C.T.C.C. n'a pas soumis de mémoire à l'Episcopat; Considérant que l'unité ouvrière au Canada ne sera possible que le jour où les locaux affiliés au Congrès du Travail du Canada se libéreront de leur affiliation aux unions américaines pour constituer un mouvement authentiquement canadien tenant compte des réalités politiques canadiennes;

Considérant que l'unité qui existe au sein du nouveau Congrès ou du Congrès unifié semble précaire étant donné que les unions vivent dans un état d'absolue indépendance les unes des autres et à l'égard de la centrale même;

Il est recommandé:

a) de prier la CTCC de soumettre à la Commission Episcopale d'Action Sociale le mémoire demandé et de ne prendre aucune décision relativement à la fusion ou à l'affiliation sans avoir au préalable reçu l'avis de l'Episcopat sur ce sujet.

b) de s'opposer à tout projet d'affiliation ou de fusion au Congrès du travail du Canada tant et aussi longtemps que les conditions énumérées ci-dessus n'auront pas été remplies. »

(1) «La CTCC et l'unité syndicale canadienne», par Gérard Dion, daus Relations Industrielles, vol. 12, no 1-2, pp. 32-55. 